DisCRETE ANALYSIS, 2018:13, 7 pp.

www.discreteanalysisjournal.com

\title{
Proof of a Conjecture of Kleinberg-Sawin-Speyer
}

\author{
Luke Pebody \\ Received 4 February 2018; Revised 25 April 2018; Published 6 July 2018
}

\begin{abstract}
In Croot, Lev and Pach's groundbreaking work [2], the authors showed that a subset of $\mathbb{Z}_{4}^{n}$ without an arithmetic progression of length 4 must be of size at most $3.1^{n}$. No prior upper bound of the form $(|G|-\varepsilon)^{n}$ was known for the corresponding question in $G^{n}$ for any abelian group $G$ containing elements of order greater than two.

Refining the technique in [2], Ellenberg and Gijswijt [3] showed that a subset of $\mathbb{Z}_{3}^{n}$ without an arithmetic progression of length 3 must be of size at most $2.755^{n}$ (no prior upper bound of the form $(3-\varepsilon)^{n}$ was known). They also provided, for any prime power $q$, a value $\lambda_{q}<q$ such that any subset of $\mathbb{Z}_{q}^{n}$ without an arithmetic progression of length 3 must be of size at most $\lambda_{q}^{n}$.

Blasiak, Church, Cohn, Grochow, Naslund, Sawin, and Umans [1] showed that the same bounds apply to tri-coloured sum-free sets, which are triples $\left\{\left(a_{i}, b_{i}, c_{i}\right): a_{i}, b_{i}, c_{i} \in \mathbb{Z}_{p}^{n}\right\}$ with $a_{i}+b_{j}+c_{k}=0$ if and only if $i=j=k$.

Building on this work, an early version of a paper by Kleinberg, Sawin and Speyer [4] gave a description of a value $\mu_{q}$ such that for any $\varepsilon>0$, tri-coloured sum-free sets of size $e^{\left(\mu_{q}-\varepsilon\right) n}$ exist in $\mathbb{Z}_{q}^{n}$ but, if $q$ is a prime power, for all sufficiently large $n$, no tri-coloured sum-free sets of size $e^{\mu_{q} n}$ exist in $\mathbb{Z}_{p}^{n}$. The value of $\mu_{q}$ was left open in the early version of this paper, but a conjecture was stated which would imply that $e^{\mu_{q}}=\lambda_{q}$, i.e. the EllenbergGijswijt bound is essentially tight for the tri-coloured sum-free set problem.

This note proves that conjecture and closes that gap. The conjecture of Kleinberg, Sawin and Speyer is true, and the Ellenberg-Gijswijt bound is essentially tight for the tri-coloured sum-free set problem.
\end{abstract}




\section{Introduction and Definitions}

Let $G$ be an abelian group. Define a tri-coloured sum-free set in $G$ to be a collection of triples $\left(a_{i}, b_{i}, c_{i}\right)$ in $G^{3}$ such that $a_{i}+b_{j}+c_{k}=0$ if and only if $i=j=k$. Write $\operatorname{sfs}(G)$ for the size of the largest tri-coloured sum-free set in $G$ and $r_{3}(G)$ for the size of the largest set in $G$ with no three-term arithmetic progression. If $X \subseteq G$ is a set with no three-term arithmetic progression, then $\{(x, x,-2 x): x \in X\}$ is a tri-coloured sum-free set, so $r_{3}(G) \leq \operatorname{sfs}(G)$.

Until recently the question of whether $\lim _{n \rightarrow \infty} r_{3}\left(G^{n}\right)^{\frac{1}{n}}$ was less than $|G|$ was open for every abelian $G$ containing elements of order greater than 2 .

Croot, Lev and Pach [2] introduced a polynomial method to show that there was indeed strict inequality for $G=\mathbb{Z}_{4}$. Building on this, Ellenberg and Gijswijt [3] showed there was strict inequality for all cyclic groups with prime power order. They proved:

Theorem 1. Let $p$ be a prime power, and let $\theta_{p}$ denote the minimum value of

$$
\frac{1+\beta+\ldots+\beta^{p-1}}{\beta^{(p-1) / 3}} .
$$

Then all sets in $\mathbb{Z}_{p}^{n}$ with no three-term arithmetic progression are of size at most $C \theta_{p}^{n}$.

Blasiak et al [1] showed that the same bounds apply to tri-coloured sum-free sets. Based on the result in this paper, Kleinberg, Sawin and Speyer [5] have shown this bound is essentially tight for tri-coloured sum-free sets, showing that:

Theorem 2. For any integer $p$ (whether a prime power or not), with $\theta_{p}$ defined as above, for $n$ sufficiently large, there are tri-coloured sum-free sets in $\mathbb{Z}_{p}^{n}$ of size at least

$$
\theta_{p}^{n} e^{-2 \sqrt{\left(2 \log \theta_{p} \log 2\right) n}-O_{p}(\log n)} .
$$

To give some motivation for the result in this paper, let $\Delta_{p}$ denote the set of distributions $\pi$ on $[p]=\{0,1,2, \ldots, p-1\}$ such that you can have three variables $X_{1}, X_{2}$ and $X_{3}$ all distributed according to $\pi$ with $X_{1}+X_{2}+X_{3}$ constant equal to $p-1$.

Given a probability distribution $\pi$ on any finite set $S$, define the entropy of $\pi$ to be

$$
\eta(\pi)=-\sum_{i \in S} \pi(i) \log \pi(i)
$$

Then if we define $\lambda_{p}=\max \left\{\eta(\pi): \pi \in \Delta_{p}\right\}$, an early version of the paper by Kleinberg, Sawin and Speyer [4] showed that:

Theorem 3. Let $p$ be a positive integer, and define $\lambda_{p}$ as above. For any $n$, if $p$ is a prime power, then all tri-coloured sum-free sets in $\mathbb{Z}_{p}^{n}$ are of size at most $e^{\lambda_{p} n}$.

For all $p$ (whether a prime power or not), for $n$ sufficiently large, there are tri-coloured sum-free sets in $\mathbb{Z}_{p}^{n}$ of size at least

$$
e^{\lambda_{p} n-2 \sqrt{\left(2 \lambda_{p} \log 2\right) n}-O_{p}(\log n)}
$$


There is a natural comparison between this and the bound of Ellenberg and Gijswijt [3]. Clearly any distribution $\pi$ in $\Delta_{p}$ has expected value $\frac{p-1}{3}$. Let $\Delta_{p}^{\prime}$ be the set of all probability distributions on $[p]$ with expected value $\frac{p-1}{3}$. Then the problem of finding $\lambda_{p}^{\prime}=\max \left\{\eta(\pi): \pi \in \Delta_{p}^{\prime}\right\}$ is considerably easier.

Let $\rho$ be the unique positive real number such that the probability distribution

$$
\psi_{\rho}(j)=\frac{\rho^{j}}{1+\rho+\ldots+\rho^{q-1}}: 0 \leq j \leq q-1
$$

has expected value $\frac{p-1}{3}$. Then $\psi_{\rho}$ is the unique distribution in $\Delta_{p}^{\prime}$ with maximal entropy. Further, this maximal entropy is precisely $\log \theta_{p}$ where $\theta_{p}$ is as defined in the statement of Theorem 1.

In the early version of their paper [4], Kleinberg, Sawin and Speyer conjectured that this distribution $\psi_{\rho}$ is in $\Delta_{p}$, and so the upper bounds in Theorem 3 and Theorem 1 are the same. The purpose of this note is to prove this conjecture.

Say that probability distributions $\pi_{1}, \pi_{2}, \pi_{3}$ are compatible if we can choose dependent random variables $X_{1}, X_{2}, X_{3}$ such that $X_{i}$ has distribution $\pi_{i}$ for each $i$ and $X_{1}+X_{2}+X_{3}$ is constant, and say that a discrete non-negative integer-valued probability distribution $\pi$ is decreasing if $\pi(0) \geq \pi(1) \geq \ldots$

We will prove the following:

Theorem 4. If three discrete non-negative integer-valued probability distributions are decreasing, only take values in $[p]=\{0,1, \ldots, p-1\}$ and have expected values summing to $p-1$ then they are compatible.

Since $\psi_{\rho}$ is clearly decreasing (as $\rho<1$ ), it will quickly follow that $\psi_{\rho}$ is in $\Delta_{p}$ thereby completing the proof of Kleinberg, Sawin and Speyer [5] that the Ellenberg-Gijswijt bound is essentially tight for the tri-coloured sum-free set problem.

\section{Simple Distributions}

In this section we will use a convexity argument to show that it will be sufficient to prove Theorem 4 if we can prove it for fairly simple distributions.

Lemma 5. Given any finite collection $\phi_{1}, \phi_{2}, \ldots, \phi_{n}$ of probability distributions on any finite set $S$, we can simultaneously express the distributions as a non-negative linear combinations $\phi_{i}=\Sigma_{j} p_{j} \phi_{i, j}$ of distributions which satisfy:

1. For each $j$, the sum of the expectations of the $\phi_{i, j}$ is the same as that of the $\phi_{i}$

2. For each $j$, at least $n-1$ of the $\phi_{i, j}$ are constant and the other (if it is not constant) takes two values.

Proof. We proceed by induction on the sum of the support sizes of the distributions. Suppose the support sizes sum to $m$, and the result is true whenever the support sizes sum to less than $m$. For $1 \leq i \leq n$, let $\min _{i}$ denote the smallest element of the support of $\phi_{i}$ and $\max _{i}$ the largest.

Clearly $\Sigma_{i} \min _{i} \leq \Sigma_{i} \mathbb{E}\left(\phi_{i}\right) \leq \Sigma_{i} \max _{i}$. Changing one of the parameters at a time over from $\min _{i}$ to $\max _{i}$ shows there exists a $k$ such that

$$
\begin{aligned}
\Sigma_{i<k} \max _{i}+\min _{k}+\Sigma_{i>k} \min _{i} & \leq \mathbb{E}\left(\phi_{1}\right)+\mathbb{E}\left(\phi_{2}\right)+\ldots+\mathbb{E}\left(\phi_{n}\right) \\
& \leq \Sigma_{i<k} \max _{i}+\max _{k}+\Sigma_{i>k} \min _{i} .
\end{aligned}
$$




\section{LUKE PEBODY}

Thus if we let $\phi_{i, 1}$ be constant equal to $\max _{i}$ for $i<k$ and constant equal to $\min _{i}$ for $i>k$ and to either be equal to $\min _{k}$ or $\max _{k}$ for $i=k$, we can rig up the probabilities to have $\Sigma_{i} \mathbb{E}\left(\phi_{i}\right)=\Sigma_{i} \mathbb{E}\left(\phi_{i, 1}\right)$.

Now if $\phi_{i}=\phi_{i, 1}$ for all $i$, we are done. Otherwise let $p_{1}$ be the smallest value of $\phi_{i}(t) / \phi_{i, 1}(t)$ (ranging over $i, t$ where the denominator is non-zero) and then $\phi_{i}^{\prime}=\frac{1}{1-p_{1}}\left(\phi_{i}(k)-p_{1} \phi_{i, 1}(k)\right)$ are distributions with the same sum of expectations but with at least one support size strictly smaller, so we can use the induction hypothesis to generate the remaining distributions.

Given an integer-valued distribution $\phi$, denote by $\widehat{\phi}$ the distribution you get by first choosing a value $i$ according to $\phi$ and then choosing a value $j$ uniformly from $\{0,1, \ldots, i\}$. Clearly $\widehat{\phi}$ is decreasing and any decreasing distribution is of this form. Furthermore, clearly this operator respects linear combinations.

We will apply this operator to the distributions in Lemma 5. It will be useful to define the distribution $U_{k}$ for each non-negative integer $k$ as the uniform distribution on $\{0,1, \ldots, k\}$ and for positive integers $k, l$ and real $x$ with $k<x<l$ the distribution $V_{k, l, x}$ as a linear combination of $\frac{l-x}{l-k}$ weight of $U_{k}$ and $\frac{x-k}{l-k}$ weight of $U_{l}$, weights so chosen as to make the expectation equal to $\frac{x}{2}$.

Corollary 6. Suppose that the positive integer $p$ satisfies that for all non-negative integers $0 \leq x_{1}, x_{2}, x_{3}<$ $p$ with $x_{1}+x_{2}+x_{3}=2(p-1), U_{x_{1}}, U_{x_{2}}$ and $U_{x_{3}}$ are compatible.

Suppose also that for all non-negative integers $0 \leq x_{1}, x_{2}, y, z<p$ with $x_{1}+x_{2}+y<2(p-1)<$ $x_{1}+x_{2}+z, U_{x_{1}}, U_{x_{2}}$ and $V_{y, z, 2 k-x_{1}-x_{2}}$ are compatible.

Then any triple of decreasing distributions on $[p]$ with expected value equal to $p-1$ are compatible.

Proof. Take such a triple $\left\{\phi_{1}, \phi_{2}, \phi_{3}\right\}$. Then as noted above, the $\phi_{i}$ can be written in the form $\widehat{\psi}_{i}$ where the $\psi_{i}$ for some distribution $\psi_{i}$ bounded above by $k$ with expected values summing to $2 k$.

By Lemma 5, $\psi_{1}, \psi_{2}, \psi_{3}$ can be expressed as a non-negative linear combination of distributions with the same sum of expected values where two are constant and the third takes at most two values.

Reapplying the operator it follows that $\phi_{1}, \phi_{2}, \phi_{3}$ can be expressed as a non-negative linear combination of distributions with the same sum of expected values where two are of the form $U_{k}$ and the third is either of the form $U_{k}$ or of the form $V_{k, l, x}$. Therefore if the assumptions of this Corollary hold, they are the linear combination of compatible distributions.

Since the non-negative linear combination of compatible distributions yields compatible distributions, it follows the original collection is compatible.

\section{The Induction Step}

Our proof of Theorem 4 will be by induction on $k$. We deal first with the case where at most one of the distributions has support including $k-1$.

Lemma 7. If $\pi_{1}, \pi_{2}, \pi_{3}$ are decreasing distributions on $[p]$ with $\mathbb{E}\left(\pi_{1}\right)+\mathbb{E}\left(\pi_{2}\right)+\mathbb{E}\left(\pi_{3}\right)=p-1$, then for all integers $t$ in the range $0 \leq t<p, \mathbb{P}\left(\pi_{1}=0\right) \leq \mathbb{P}\left(\pi_{2}>t\right)+\mathbb{P}\left(\pi_{3} \geq(p-1)-t\right)$.

Proof. If $\pi$ is a decreasing distribution on $[p]$ then for any integer $0 \leq t<p$, the expected value of $\pi$ given $\pi>t$ is at most $\frac{t+p}{2}$ and the expected value of $\pi$ given $\pi \leq t$ is at most $\frac{t}{2}$.

Thus the expected value of $\pi$ is at most $\frac{t}{2}+\frac{p}{2} \mathbb{P}(\pi>t)$. 
Therefore

$$
\begin{aligned}
p-1 & =\mathbb{E}\left(\pi_{1}\right)+\mathbb{E}\left(\pi_{2}\right)+\mathbb{E}\left(\pi_{3}\right) \\
& \leq \frac{0+t+(p-2)-t}{2}+\frac{p}{2}\left(\mathbb{P}\left(\pi_{1}>0\right)+\mathbb{P}\left(\pi_{2}>t\right)+\mathbb{P}\left(\pi_{3}>p-2-t\right)\right) \\
& \leq \frac{p-2}{2}+\frac{p}{2}\left(\mathbb{P}\left(\pi_{1}>0\right)+\mathbb{P}\left(\pi_{2}>t\right)+\mathbb{P}\left(\pi_{3}>p-2-t\right)\right)
\end{aligned}
$$

which simplifies to $1 \leq \mathbb{P}\left(\pi_{1}>0\right)+\mathbb{P}\left(\pi_{2}>t\right)+\mathbb{P}\left(\pi_{3}>p-2-t\right)$. Subtracting $\mathbb{P}\left(\pi_{1}>0\right)$ from both sides gives the required inequality.

This allows us to handle a lot of cases by induction.

Corollary 8. Suppose that for all decreasing distributions $\pi_{1}, \pi_{2}, \pi_{3}$ on $[p-1]$ with $\mathbb{E}\left(\pi_{1}\right)+\mathbb{E}\left(\pi_{2}\right)+$ $\mathbb{E}\left(\pi_{3}\right)=p-2, \pi_{1}, \pi_{2}$ and $\pi_{3}$ are compatible.

Then for all decreasing distributions $\pi_{1}, \pi_{2}, \pi_{3}$ on $[p]$ with $\mathbb{E}\left(\pi_{1}\right)+\mathbb{E}\left(\pi_{2}\right)+\mathbb{E}\left(\pi_{3}\right)=p-1$ with $\pi_{2}(p-1)=\pi_{3}(p-1)=0, \pi_{1}, \pi_{2}$ and $\pi_{3}$ are compatible.

Proof. Clearly $\pi_{2}(0)>\pi_{3}(p-1)$ and $\pi_{2}(p-1)<\pi_{3}(0)$, so there must exist an integer $t$ in the range $0 \leq t<p$ such that $\pi_{2}(k)>\pi_{3}((p-1)-k)$ for $k \leq t$ and $\pi_{2}(k) \leq \pi_{3}((p-1)-k)$ for $k>t$.

This means that

$$
\Sigma_{k \leq t} \min \left(\pi_{2}(k), \pi_{3}((p-1)-k)\right)=\Sigma_{k \leq t} \pi_{3}((p-1)-k)=\mathbb{P}\left(\pi_{3} \geq p-1-t\right)
$$

and

$$
\Sigma_{k>t} \min \left(\pi_{2}(k), \pi_{3}((p-1)-k)\right)=\Sigma_{k>t} \pi_{2}(k)=\mathbb{P}\left(\pi_{2}>t\right) .
$$

By Lemma 7 , it follows that $\mathbb{P}\left(\pi_{1}=0\right) \leq \Sigma_{k} \min \left(\pi_{2}(k), \pi_{3}((p-1)-k)\right)$. Thus there exists a real number $x$ such that

$$
\mathbb{P}\left(\pi_{1}=0\right)=\Sigma_{k} \min \left(\pi_{2}(k), \pi_{3}((p-1)-k), x\right) .
$$

For $0 \leq k \leq p-1$, let $f(k)=\min \left(\pi_{2}(k), \pi_{3}((p-1)-k), x\right)$. Since $\min \left(\pi_{2}(k), x\right)$ is non-increasing, if $f(k)<f(k+1), f(k)=\pi_{3}((p-1)-k)$. Similarly, if $f(k)>f(k+1), f(k+1)=\pi_{2}(k+1)$.

Therefore if we define $g_{2}(k)=\pi_{2}(k)-f(k)$, either $f(k) \leq f(k+1)$, and hence $g_{2}(k) \geq g_{2}(k+1)$, or $f(k)>f(k+1)$, in which case $g_{2}(k) \geq 0=g_{2}(k+1)$. Thus $g_{2}$ is non-increasing. Similarly, so is $g_{3}(k)=\pi_{3}(k)-f((p-1)-k)$.

If we start defining our dependent random variables by saying that with probability $f(k), X_{1}=0$, $X_{2}=k$ and $X_{3}=(p-1)-k$, the remaining distribution on $\pi_{1}$ is non-increasing on $\{1, \ldots, p-1\}$ and the remaining distributions on $\pi_{2}$ and $\pi_{3}$ are non-increasing on $\{0, \ldots, p-2\}$.

Thus if we subtract 1 from the remaining distribution on $\pi_{1}$, we are left with decreasing distributions on $\{0, \ldots, p-2\}$ with expected values that sum to $p-2$, which are compatible. It follows that $\pi_{1}, \pi_{2}$ and $\pi_{3}$ must be compatible. 
LUKE PEBODY

\section{The remaining case}

Corollary 8 is enough to cover almost all cases of the assumptions of Corollary 6. In this section we will deal with the remaining case.

Lemma 9. For any non-negative integers $m$ and $n$ one can pair distributions $X$ and $Y$ that are uniform on $\{0,1, \ldots, m\}$ and $\{0,1, \ldots, n\}$ such that $X+Y$ is uniform on $\{0,1, \ldots, m+n\}$.

Proof. Let $A_{1}, \ldots, A_{m}, B_{1}, \ldots, B_{n}$ and $C$ be independent identically distributed random variables with continuous distributions. Denote $\left|i: A_{i}<C\right|$ by $X$ and $\left|j: B_{j}<C\right|$ by $Y$. Clearly $X$ is uniform on $\{0,1, \ldots, m\}, Y$ is uniform on $\{0,1, \ldots, n\}$ and $X+Y$ is uniform on $\{0,1, \ldots, m+n\}$.

Combining two instances of this lemma together gives the following.

Corollary 10. For any integers $i$ and $j$ with $i>0, j \geq 0$ and $i+j<p-1$, the distributions $U_{i}, U_{p-1}$ and $V_{j, p-1, p-1-i}$ are compatible.

Proof. Firstly flip a coin which lands on heads with probability $\frac{i+j+1}{p}$.

If the coin comes up heads, use Lemma 9 to sample random variables $X$ and $Y$ where $X$ is uniform on $\{0,1, \ldots, i\}, Y$ is uniform on $\{0,1, \ldots, j\}$ and $X+Y$ is uniform on $\{0,1, \ldots, i+j\}$. Then let $X_{1}=$ $X, X_{2}=(p-1)-X-Y$ and $X_{3}=Y$.

If the coin comes up tails, use Lemma 9 to sample random variables $X^{\prime}$ and $Y^{\prime}$ where $X^{\prime}$ is uniform on $\{0,1, \ldots, i\}, Y^{\prime}$ is uniform on $\{0,1, \ldots, p-(i+j+2)\}$ and $X^{\prime}+Y^{\prime}$ is uniform on $\{0,1, \ldots, p-(j+2)\}$. Then let $X_{1}=X^{\prime}, X_{2}=Y^{\prime}$ and $X_{3}=(p-1)-X^{\prime}-Y^{\prime}$.

$X_{1}$ is uniform on $\{0,1, \ldots, i\}$ regardless of the coin-flip. $X_{2}$ is uniform on $\{0,1, \ldots, p-(i+j+2)\}$ with probability $\frac{p-(i+j+1)}{p}$ and uniform on $\{p-(i+j+1), \ldots, p-1\}$ with probability $\frac{i+j+1}{p}$, so is uniform on $\{0, \ldots, p-1\}$.

Finally $X_{3}$ is a combination of uniform on $\{0,1, \ldots, j\}$ and uniform on $\{j+1, \ldots, p-1\}$ with mean $\frac{p-1-i}{2}$, so it must be $V_{j, p-1, p-1-i}$.

\section{Putting it all together}

We now have all of the ingredients for the proof of Theorem 4.

Proof of Theorem 4. We proceed by induction on $p$.

If $p=1, \pi_{1}, \pi_{2}$ and $\pi_{3}$ are constant equal to 0 and are trivially compatible.

Now suppose that Theorem 4 is true for $p-1$, and we will attempt to prove it for $p$. By Corollary 6 , it is sufficient to prove that $U_{k}, U_{l}$ and $U_{m}$ are compatible when $0 \leq k, l, m<p$ and $k+l+m=2(p-1)$ and $U_{k}, U_{l}$ and $V_{m, n, 2(p-1)-k-l}$ are compatible when $0 \leq k, l, m, n<p$ with $k+l+m<2(p-1)<k+l+n$.

By Corollary 8, the induction hypothesis implies it is true for all such $U_{k}, U_{l}$ and $U_{m}$ if two of $k, l$ and $m$ are less than $p-1$ and for all such $U_{k}, U_{l}$ and $V_{m, n, 2(p-1)-k-l}$ if two of $k, l$ and $n$ are less than $p-1$.

So, for $U_{k}, U_{l}$ and $U_{m}$ we can assume that at least two of $k, l$ and $m$ are equal to $p-1$. Since $k+l+m=2(p-1)$, it follows that two are uniform on $[p]$ and the other is constant equal to 0 . Then these are compatible: let $X_{1}=0, X_{2}$ be uniform on $[p]$ and let $X_{3}=(p-1)-X_{2}$. 
Similarly, for $U_{k}, U_{l}$ and $V_{m, n, 2(p-1)-k-l}$, we can assume that at least two of $k, l$ and $n$ are equal to $p-1$. Since $k+l+m<2(p-1)$, it follows that $n$ must equal $p-1$ and one of the others must be equal to $p-1$, so they are of the form stated in Corollary 10 .

Finally, since the distribution $\psi_{\rho}$ is clearly decreasing (as $\mathbb{E} \psi_{\rho}=\frac{p-1}{3}<\frac{p-1}{2}$, and hence $\rho<1$ ), it follows from Theorem 4 that $\psi_{\rho}$ is compatible with two copies of itself, which means that $\psi_{\rho} \in \Delta_{p}$, completing the proof by Kleinberg-Sawin-Speyer that the Ellenberg-Gijswijt bound is essentially tight for tri-coloured sum-free sets.

\section{References}

[1] Jonah Blasiak, Thomas Church, Henry Cohn, Joshua A. Grochow, Eric Naslund, William F. Sawin, and Chris Umans. On cap sets and the group-theoretic approach to matrix multiplication. Discrete Analysis, 2017:3 27pp 1,2

[2] Ernie Croot, Vsevolod Lev and Peter Pach. Progression-free sets in $\mathbb{Z}_{4}^{n}$ are exponentially small. Ann. of Math., 185(1):333-337, 2017 1, 2

[3] Jordan S. Ellenberg and Dion Gijswijt. On large subsets of $F_{q}^{n}$ with no three-term arithmetic progression Ann. of Math., 185(1):339-343, 2017 1, 2, 3

[4] Robert Kleinberg, William F. Sawin, and David E. Speyer. The growth rate of tri-colored sum-free sets (preprint). 2017, arXiv:1607.00047v1 1, 2, 3

[5] Robert Kleinberg, William F. Sawin, and David E. Speyer. The growth rate of tri-colored sum-free sets. Discrete Analysis, 2018:12, 10pp 2, 3

\section{AUTHOR}

Luke Pebody

Rokos Capital Management

23 Savile Row

London, United Kingdom

luke [at] pebody [dot] org 